\title{
Modelling social interaction between humans and service robots in large public spaces
}

\author{
Bani Anvari ${ }^{1,2}$ and Helge A Wurdemann ${ }^{2}$
}

\begin{abstract}
With the advent of service robots in public places (e.g., in airports and shopping malls), understanding sociopsychological interactions between humans and robots is of paramount importance. On the one hand, traditional robotic navigation systems consider humans and robots as moving obstacles and focus on the problem of real-time collision avoidance in Human-Robot Interaction (HRI) using mathematical models. On the other hand, the behavior of a robot has been determined with respect to a human. Parameters for human-human interaction have been assumed and applied to interactions involving robots. One major limitation is the lack of sufficient data for calibration and validation procedures.

This paper models, calibrates and validates the sociopsychological interaction of the human in HRIs among crowds. The mathematical model is an extension of the Social Force Model for crowd modelling. The proposed model is calibrated and validated using open source datasets (including uninstructed human trajectories) from the Asia and Pacific Trade Center shopping mall in Osaka (Japan).

In summary, the results of the calibration and validation on the multiple HRIs encountered in the datasets show that humans react to a service robot to a higher extend within a larger distance compared to the interaction range towards another human. This microscopic model, calibration and validation framework can be used to simulate HRI between service robots and humans, predict humans' behavior, conduct comparative studies, and gain insights into safe and comfortable humanrobot relationships from the human's perspective.
\end{abstract}

\section{INTRODUCTION}

The deployment of autonomous service robots assisting human beings has gained huge popularity in recent years. The areas of application initially included transportation of goods, e.g., linen, laboratory samples, medicine in hospitals [1]-[3], delivering items such as documents, drinks and meals in multi-floor offices [4], as well as collecting waste, and delivering food in cities [5]. Over the years, this list was extended to tasks involving other types of interactions with humans including assisting disabled people, performing security patrols, guiding visitors in museums, and entertaining people [6]-[10]. Robots have proven themselves to be effective at providing service to Frequently-Asked Questions (FAQs) such as giving direction, distributing information leaflets and helping customers to find their way in shopping malls (i.e., repetitive, monotonous service tasks) [11], [12]. Human-Robot Interaction (HRI) has been experimentally

\footnotetext{
${ }^{1}$ Bani Anvari is with the Transportation Research Group, Civil, Maritime and Environmental Engineering and Science, Boldrewood Innovation Campus, University of Southampton, UK

${ }^{2}$ Bani Anvari is with the Centre for Transport Studies, Department of Civil, Environmental and Geomatic Engineering and Helge A Wurdemann is with the Department of Mechanical Engineering, University College London, UK. b.anvari@ucl.ac.uk
}

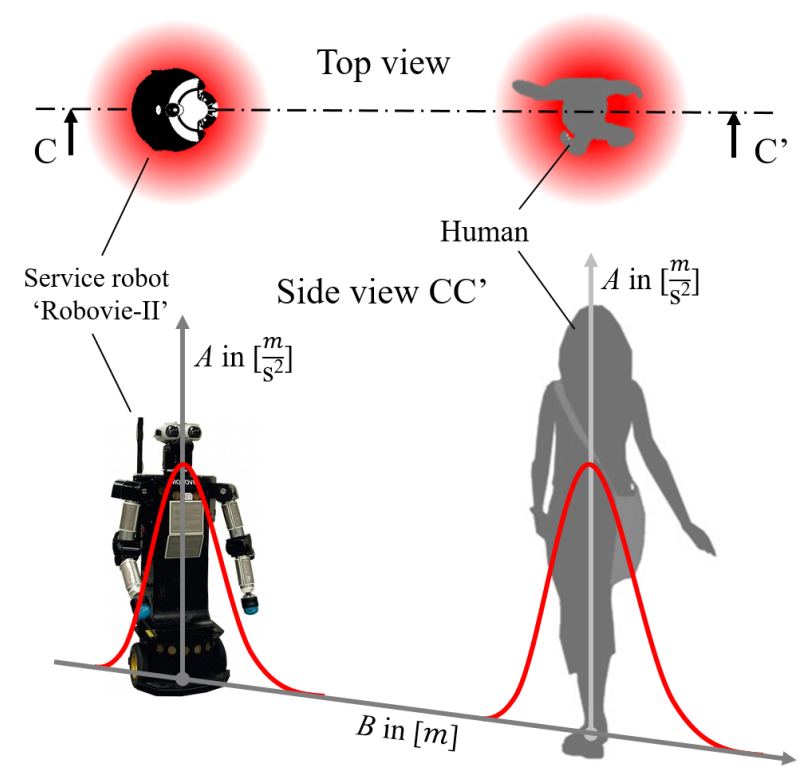

Fig. 1. Modeling Human-Robot Interaction based on the Social Force Model: Parameter $A$ is the interaction strength which represents the amount of influence that a force has and depends on the interaction range $B$.

studied within various environments (e.g., an office, a domestic environment, a seminar room) and for different tasks [13]-[19]. During social interactions, humans tend to keep a measurable distance from their counterpart: In $60 \%$ of the observed cases, this distance does not differ between a robotic or human counterpart; in $40 \%$ of the observed cases, the distance is less, if the counterpart is a robot [13], [14]. Further studies have shown that the most comfortable direction for a robot to approach a human is from either the left or right hand side [17]-[19]. It is also found that humans prefer a robot in the shared environment to move at a speed lower than human walking speed (lower than $1 \frac{\mathrm{m}}{\mathrm{s}}$ ) [20]. The correlated relationship between speed, direction of approach and distance in HRI is of paramount importance ensuring human's safety and comfort [21].

Safety in HRI, where humans share space with service robots, is commonly assured by avoiding collisions with humans. Collision-free navigation of robots among humans is modelled in various studies where humans are considered as dynamic obstacles [22]-[25]. A method is proposed in [21] to determine an optimal trajectory for robot's motion among humans. They define distance-dependent velocities for HRI to assure human's safety and comfort. However, human motion is self-driven and self-adapted, and it is 
subject to both physical laws and psychological principles. In [26], this has been realised by developing a social force based model for motion control of mobile robots in shared environments with humans. The model was tested in two scenarios: collision-avoidance of a robot with a static human and human-following behavior of a robot without entering the human's intimate zone. Validation was performed using experimental tests defined with the Virtual Robot Experimentation Platform only. In [27], the social behavior of a robot is modelled based on the Social Force Model (SFM) to walk side-by-side a human in a crowd and make the human feel safe and comfortable. The authors extended the SFM for this purpose since it has the capability of replicating human behaviors in crowds accurately. In [28], the role of haptic feedback for human-robot communication is tested for an orthogonal human-robot encounter. Trajectories of human movements are predicted using the SFM as well. Accordingly, the robots's intention of movement is communicated to the human prior to their interaction using a wearable haptic device. It is reported that haptic feedback supports humans to feel safe around robots and improve the humanrobot relationship. However, the authors mention that the social force based model is not accurate and their interaction scenarios are very limited (i.e., only orthogonal encounters are considered).

The limitations of the above mentioned studies are manifold: (i) HRI and collision avoidance behavior of a service or mobile robot have been explored with respect to a human only; (ii) Some studies have applied the SFM and its parameters for human-human interaction to interactions involving robots; (iii) An extended SFM has been calibrated for orthogonal encounters between humans and robots only; and (iv) The proposed models are not calibrated and validated using uninstructed pedestrian trajectories in real-life scenarios.

This paper focuses on modelling, calibrating and validating the socio-psychological interaction of the human in HRIs (the human-robot interaction rather than the robot-human interaction) among crowds in a shopping mall. As illustrated in Figure 1, the social interaction is modelled based on an extended version of the Social Force Model (SFM) in this study. The SFM is a mathematical model which was initially proposed for crowd simulation [29]. The social HRI is modelled based on our previous work describing the interaction between pedestrians and vehicles [30]-[34]. In this paper, real-world datasets obtained from the Asia and Pacific Trade Center (ATC) shopping mall in Osaka (Japan) [35], [36] are used to calibrate and validate the extended version of the SFM.

Section II gives an overview of the case study in the ATC shopping mall in Osaka (Japan) and describes the recorded scenarios and available data sets. The SFM is modified and applied to capture the social interaction between humans and service robots in Section III. The calibration and validation procedures including the interpretation of the results are described in Section IV-B. Section V summaries the achievements of this paper and outlines future work and practical applications.

\section{BACKGROUND ON OPEN SOURCE DATA OF THE ATC SHOPPING MALL CASE STUDY, OSAKA, JAPAN}

\section{A. Deploying service robots in a large public space}

As part of a Japan Science and Technology Agency (JST/CREST) funded project on enabling mobile social robots to work in public spaces [35]-[37], a service robot was placed within the ATC shopping mall in Osaka (Japan); time-dependent positions of humans were tracked using 3D range sensors and HRIs were monitored within a certain area.

As shown in Figure 2, part of the shopping mall's area includes a large hall on the west side which leads to a corridor on the east side with a number of shops alongside [35]. The east side corridor leads to a train station and the remaining area of the ATC shopping mall. On the north side of the ATC shopping mall, there are slopes, escalators and elevators which lead to a number of shops, offices and parking lots. On the west side of the large hall, a ticket office is located followed by an exit towards a ferry terminal. On the south side, there are stairs to exit the building. The stair cases in the centre of the map in Figure 2 allow people to reach the third floor of this building. The main entry and exit areas (yellow) and the location of the service robot (blue) are shown in Figure 2. The solid colorful lines in the middle represent the tracked trajectories of humans in the ATC shopping mall over a duration of $1.5 \mathrm{hr}$ (6:00pm-7:30pm, Japan Standard Time (JST)).

The service robot Robovie-II (see Figure 1) was placed in this shopping mall which is a $120 \mathrm{~cm}$ tall robot with a circular footprint of $0.3 \mathrm{~m}$ radius. The robot's head has 3 Degrees of Freedom (DoFs); the arms have 4 DoFs. RobovieII was dressed in a staff uniform/arm band with a front signboard stating "Information Staff". The service robot was programmed to move back and forth on a predefined path, and to react to peoples responses. Two laser range sensors used to avoid physical collision, a microphone and speakers allowing verbal interaction with people were mounted on the robot. The linear and angular speed of the robots were

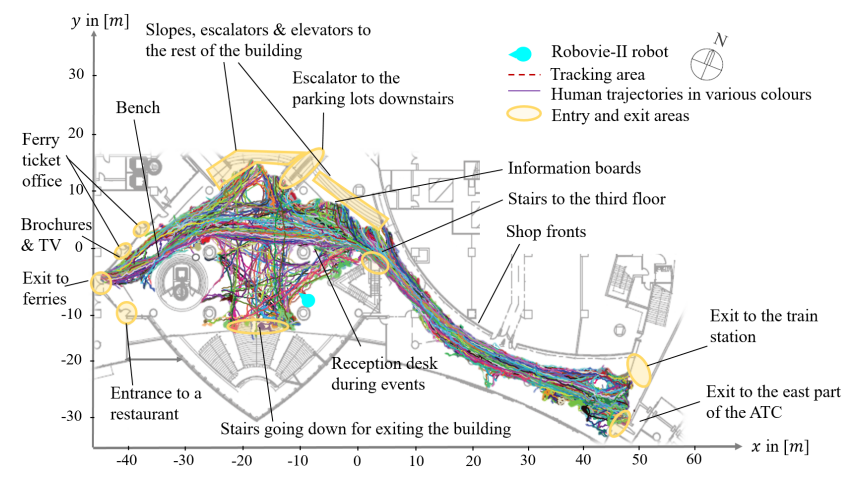

Fig. 2. Tracking area within the ATC shopping mall in Osaka (Japan). The yellow areas indicate its entry and exit points. The solid colourful lines in the middle indicate human trajectories over a duration of $1.5 \mathrm{hr}$ $(6: 00-7: 30 \mathrm{pm}($ JST) $)$. 
set to $0.6 \frac{\mathrm{m}}{\mathrm{s}}$ and $42^{\circ} / \mathrm{s}$, respectively. The linear and angular acceleration of the robot were set to $0.6 \frac{\mathrm{m}}{\mathrm{s}^{2}}$ and $30^{\circ} / \mathrm{s}^{2}$. A tracking system consisting of forty-nine 3-D range sensors was set up on the ceiling of the ATC shopping mall in order to track human locations and monitor HRIs every $33 \mathrm{~ms}$. More information about Robovie-II and the tracking system can be found in [35].

\section{B. Description of scenarios and available open source data}

Using the tracking system installed in the ATC shopping centre, data including velocities, Cartesian coordinates, angle of motions, and facing angles of humans and the robot were collected over time. This information is available in two open source datasets:

1) One dataset includes the above information of the ATC shopping mall's visitors between 24/10/2012 and $29 / 11 / 2013$ [35]. The tracking system covered $900 \mathrm{~m}^{2}$ of the shopping mall and was collected over 92 days' worth of data with up to 40 million entries.

2) Another dataset captured the information of humans when in interaction with the robot allocated in the the shopping mall's large hall (see the location in Figure 2) [36]. This dataset includes 63 scenarios where a human intended to initiate an interaction (e.g., seek help) with Robovie-II, and 67 scenarios where a human was not interested in any interaction with the service robot at first, but started interacting when Robovie-II proactively moved towards him/her. All scenarios are $10 \mathrm{~s}$ long, ending either when the human reached the closest point to Robovie-II or when the human started a request. This dataset contains information about the service robot and humans who are interacting with it and also data on all other humans who were close-by at the considered moment in time.

\section{Ethics}

Since the ATC shopping mall datasets were collected by a third party who made anonymised data available to the public to use for research purposes, a permission to perform secondary data analysis was obtained from the Ethics Department of the University of Southampton (Ref. 40418) which took into account the datasets used, the type of analysis carried out and the risks involved.

\section{APPLIED SOCIAL FORCE MODEL TO HUMAN-ROBOVIE-II INTERACTIONS}

\section{A. Recalling the Social Force Model}

The Social Force Model (SFM) is one of the most accredited models for pedestrian simulation [38]. The concept is based on Lewin's field theory in social science that behavioral changes are driven and self-possessed by social fields [39]. Helbing formulated this concept in a mathematical format in 1991 and defined pedestrian movements based on the influence of external psychological forces exerted by other humans and repulsive forces imposed from obstacles [29]. The main forces in the SFM are as follows (see Figure 3) [40]:
- The driving force $\vec{f}_{\alpha}^{0}$ persuades human $\alpha$ to move towards the desired direction $\vec{e}_{\alpha}^{0}$ with the desired speed $v_{\alpha}^{0}$ that is adapted to the actual velocity $\vec{v}_{\alpha}$ within a relaxation time $\tau_{\alpha}$. The difference between the desired velocity and actual velocity takes into account the human's subjective desire as explained in Equation 1.

$$
\vec{f}_{\alpha}^{0}=\frac{v_{\alpha}^{0} \vec{e}_{\alpha}^{0}(t)}{\tau_{\alpha}}-\frac{\vec{v}_{\alpha}^{0}}{\tau_{\alpha}}
$$

- The interaction force $\vec{f}_{\alpha \beta}$ keeps a desired distance from another human in their social relationship. This force is the sum of two forces exerted from closeby human $\beta$ to human $\alpha$ as expressed in Equation 2 . The social interaction force $\vec{f}_{\alpha \beta}^{\text {soc }}$ reflects the sociopsychological behavior of a human to keep a desired distance from human $\beta$. This is explained using an exponential function in Equation 3. Here, $A_{\alpha \beta}$ and $B_{\alpha \beta}$ describe the interaction strength and the interaction range respectively. $\vec{n}_{\alpha \beta}$ is the normalised vector pointing from $\beta$ to $\alpha . F_{\alpha \beta}$ is the form factor, which reflects the fact that movements within the field of view of a human can persuade him/her to react more compared to movements behind him/her.

The physical interaction force $\vec{f}_{\alpha \beta}^{p h}$ represents the pressure exerted from other humans in case of physical contact, and the sliding friction force imposed to a human in high density conditions. The physical interaction force is valid only when there is a physical contact.

$$
\begin{gathered}
\vec{f}_{\alpha \beta}(t)=\vec{f}_{\alpha \beta}^{s o c}(t)+\vec{f}_{\alpha \beta}^{p h}(t) \\
\vec{f}_{\alpha \beta}^{s o c}=A_{\alpha \beta} e^{\frac{r_{\alpha \beta}-d_{\alpha \beta}}{B_{\alpha \beta}}} \vec{n}_{\alpha \beta} F_{\alpha \beta}
\end{gathered}
$$

- The interaction force $\vec{f}_{\alpha b}$ maintains a distance from obstacle $b$ and models any social and physical interaction presented in Equation 4. These forces are imposed from a nearby obstacle $b$ to human $\alpha$ as shown in Figure 3 .

$$
\vec{f}_{\alpha b}(t)=\vec{f}_{\alpha b}^{s o c}(t)+\vec{f}_{\alpha b}^{p h}(t)
$$

- The fluctuation force $\vec{\xi}$ replicates the variation of human beings behavior. The diverse behaviors of humans cannot be measured and are presented with a force that is Gaussian distributed and perpendicular to the desired direction of movement (see Figure 3).

The sum of all forces above persuades humans' movement and direction. In this model, psychological factors are presented in parameters such as desired velocity and social forces which are not physical entities since they describe people's preferences or certain characteristics of human mind. In addition, contrary to Newtons third law of motion, a pair of forces acting on two interacting agents (e.g., a human and another human) are not equal here and they depend on a number of parameters such as interaction range, interaction strength, field of view. The detailed mathematical format of these forces can be found in [39]-[41]. 


\section{B. Overview of the new Social Force Model}

In this study, humans are modelled based on the original SFM [39]-[41]. The existence of the service robot Robovie-II in the shopping mall is expressed by a new interaction force $\vec{f}_{\alpha r}$ exerted to human $\alpha$ from robot $r$ in addition to the forces from nearby humans $\beta$ and obstacles $b$ (see Figure 3). The sum of all the forces is used to describe human's behavior in the ATC shopping mall as shown in Equation 5:

$$
\frac{d \vec{v}_{\alpha}(t)}{d t}=\vec{f}_{\alpha}^{0}+\sum_{\beta} \vec{f}_{\alpha \beta}+\sum_{r} \vec{f}_{\alpha r}+\sum_{b} \vec{f}_{\alpha b}+\vec{\xi}
$$

The detailed mathematical format of the new interaction force is explained in Section III-C.

\section{Interaction between humans and service robots}

The new interaction force $\vec{f}_{\alpha r}$ is defined in Equation 6 and consist of two forces $\vec{f}_{\alpha r}^{s o c}$ and $\vec{f}_{\alpha r}^{p h}$. The socio-psychological force $\vec{f}_{\alpha r}^{s o c}$ keeps a certain distance from the nearby robot $r$. The physical interaction force $\vec{f}_{\alpha r}^{p h}$ replicates any physical contact between human $\alpha$ and robot $r$.

$$
\vec{f}_{\alpha r}(t)=\vec{f}_{\alpha r}^{s o c}(t)+\vec{f}_{\alpha r}^{p h}(t)
$$

$f_{\alpha r}^{p h}$ is only valid for physical contact between robot $r$ and human $\alpha$. Since no physical contact was reported in the second dataset, the physical force is set to zero in this study. The mathematical format of the social interaction force between human $\alpha$ and robot $r$ can be seen in Equation 7:

$$
\vec{f}_{\alpha r}^{s o c}=A_{\alpha r} e^{\frac{r \alpha r-d \alpha r}{B \alpha r}} \vec{n}_{\alpha r} F_{\alpha r}
$$

where,

$$
\begin{gathered}
r_{\alpha r}=r_{\alpha}+r_{r} \\
F_{\alpha r}=\lambda_{\alpha}+\left(1-\lambda_{\alpha}\right) \frac{1+\cos \left(\phi_{\alpha r}\right)}{2}
\end{gathered}
$$

Similar to the interaction force between two humans in the SFM, an exponential function is applied to human $\alpha$ to

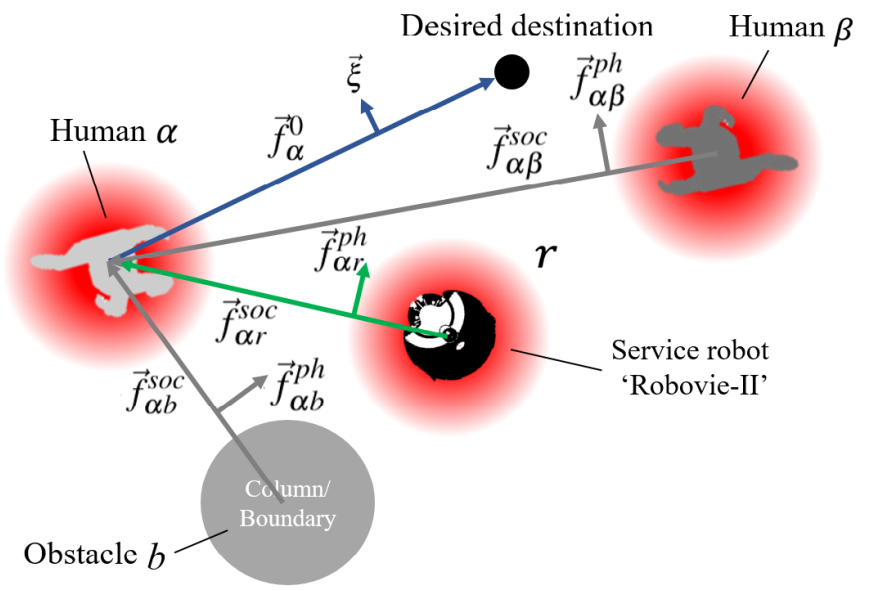

Fig. 3. Force terms exerted to human $\alpha$ from a close-by human $\beta$, robot $r$, and obstacle $b$. The forces in green are new terms introduced in this study for taking into account the socio-psychological behavior between humans and service robots. capture the influence of distance between human $\alpha$ and the nearby robot $r$. A circle with radius $r_{a}$ is defined for a human and a circle with radius $r_{r}$ is defined for a robot. $d_{\alpha r}$ is the distance between the centre of mass of human $\alpha$ and robot $r . \vec{n}_{\alpha r}$ is the normalized vector pointing from robot $r$ to human $\alpha$.

$A_{\alpha r}, B_{\alpha r}$ are positive constant parameters that will be determined during the calibration process. $A_{\alpha r}$ is called interaction strength which represents the amount of influence that the interaction force has on human $\alpha$ and describes the amplitude of the force exerted from robot $r$. $A_{\alpha r}$ depends on the interaction range $B_{\alpha r}$ which can be interpreted as the distance that human beings react (decelerate) when moving towards a robot. As a result, a close distance will contribute to a considerable high deceleration to avoid collision. $B_{\alpha r}$ effects how quickly the exerted force decreases with distance. $F_{\alpha r}$ is a form factor to replicate the anisotropic character of human interactions. $F_{\alpha r}$ has a dimpled limacon shape depending on the value of $0 \leqslant \lambda_{\alpha} \leqslant 1$ and $\phi_{\alpha r}$. The value of $\lambda_{\alpha}$ implies that impact of a human in front is larger than behind. $\phi_{\alpha r}$ is the angle between the desired direction of movement of human $\alpha$ and the direction of the repulsive force exerted by robot $r$. As a result of $F_{\alpha r}$, humans are more influenced by robot $r$ 's behavior when approaching from the front.

\section{CALIBRATION, VALIDATION AND RESULTS}

As mentioned in Section III-B, three type of interaction forces are represented in the proposed force-based model: the human-human interaction force, the human-obstacle interaction force and the human-robot interaction force. The first two interaction forces have been studied in [33], [41]. Hence, we will calibrate and validate the human-robot interaction parameters $\left(A_{\alpha r}\right.$ and $\left.B_{\alpha r}\right)$ in this section.

\section{A. Data analysis of the two available datasets}

Statistical analysis is performed on the two datasets (see Section II-B) to understand the humans' speed, acceleration and their direction when in interaction with the service robot Robovie-II inside the ATC shopping mall. Figure 4 shows the results with regards to the speed and acceleration of shopping mall visitors. The mean speed and acceleration of humans in the ATC shopping centre is observed to be

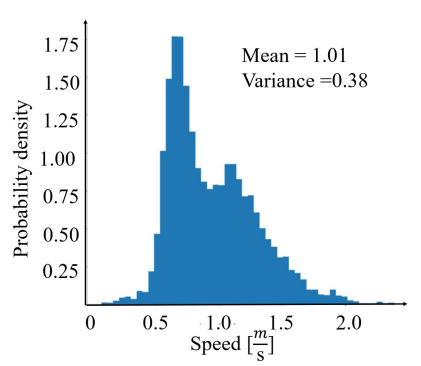

(a)

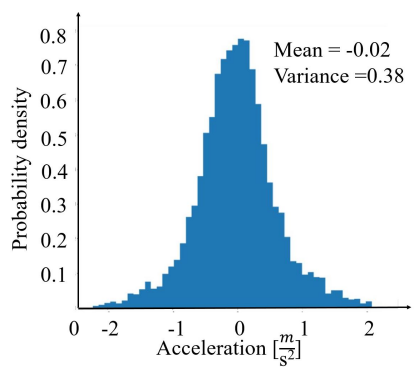

(b)
Fig. 4. (a) Speed histogram (mean value of $1.01 \frac{\mathrm{m}}{\mathrm{s}}$ and variance of 0.38 ), (b) Acceleration histogram (mean value of $-0.02 \frac{\mathrm{m}}{\mathrm{s}^{2}}$ and variance of 0.38 ) for visitors of the ATC shopping mall over 1.5 hours. 
$1.01(\sigma=0.38) \frac{\mathrm{m}}{\mathrm{s}}$ and $-0.02(\sigma=0.38) \frac{\mathrm{m}}{\mathrm{s}^{2}}$. The mean speed of humans in human-robot interaction scenarios were within a similar range of $1.25(\sigma=0.1) \frac{\mathrm{m}}{\mathrm{s}}$. The latter mean value is used in the calibration (Section IV-B) and validation (Section IV-C) procedures as the desired speed of the human when interacting with the robot.

\section{B. Calibration process of the extended SFM}

A calibration process is applied to determine the optimal values for interaction parameters $\left(A_{\alpha r}\right.$ and $\left.B_{\alpha r}\right)$ in order to be able to replicate real world trajectories. About $70 \%$ of the dataset is allocated for calibration and the remaining for validation. As shown in Figure 5, a hybrid method using empirical and simulated trajectories is used for the calibration of these parameters. From the available tracking information, a number of scenarios with human-robot interactions are selected. For each human, a virtual agent is assigned in the simulation domain developed in [32]. A simulation is initiated according to real data in which one agent (human) is moving with the new mathematical model while the other agents (other humans or the robot) are moving according to the extracted trajectories. For instance, the dark grey agent/human and the robot in the synthetic example in Figure 5(a) follow extracted trajectories. The light grey agent/human moves according to the force directed model. Once these are set, the simulated trajectory (dashed line) is compared to the empirical trajectory (solid line) for

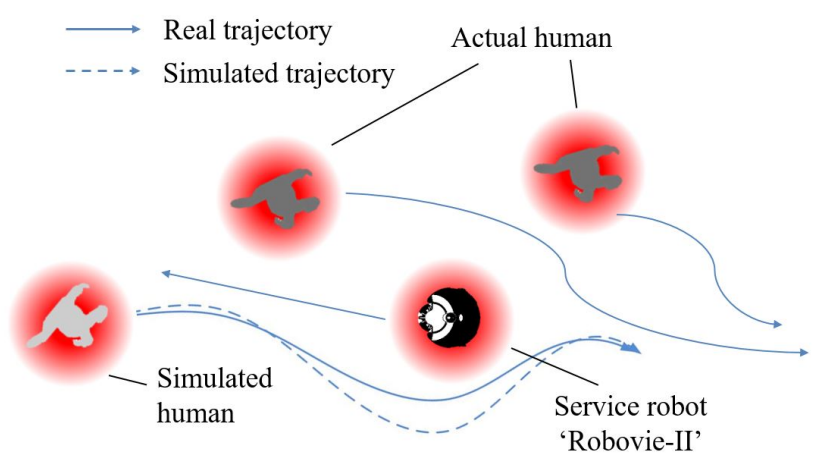

(a)

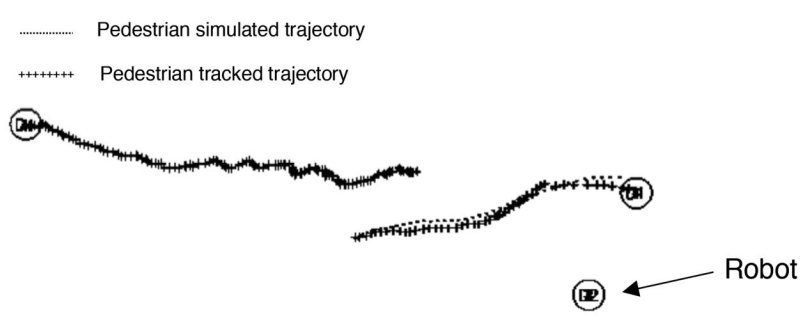

(b)

Fig. 5. (a) Synthetic illustration of the hybrid calibration method. The light grey human moves based on the extended SFM whereas other agents follow the extracted trajectories obtained from the two available datasets. (b) One of the simulation results using this method. The real world trajectory is shown with a (+++) line and the simulated trajectory using the extended SFM is presented using a dotted line. different combination values of parameters $\left(A_{\alpha r}\right.$ and $\left.B_{\alpha r}\right)$ until the best fitness value is determined. An example of this calibration process is demonstrated in Figure 5(b).

There are three common approaches to assess how matching the simulation results are with respect to the observed data. The first one is the acceptable window which compares the results of a model to observed ranges for each endpoint and does not include the degree of closeness. The second approach is the likelihood function where a distribution is produced by minimising the square distance between real and simulated data. The third method is minimising deviation between real and simulated data by applying different parameter sets and measuring the magnitude of goodness of fit. The third method is applied in this paper to allow differentiating all the data points continuously and within a short time frame.

The hybrid calibration method proposed in [42] is applied to minimise the deviations between the real and simulated trajectory of a human when interacting with the robot and other humans. The best combination of parameters for the interaction strength $A_{\alpha r}$ and interaction range $B_{\alpha r}$ is the one that results in the minimum average relative distance error $E$ in Equation 8.

$$
E=\frac{\left\|r_{\alpha}^{\text {simulated }}(t+T)-r_{\alpha}^{\text {tracked }}(t+T)\right\|}{\left\|r_{\alpha}^{\text {simulated }}(t+T)-r_{\alpha}^{\text {tracked }}(t)\right\|}
$$

Here, $t$ is the starting time of a tracked trajectory, $T$ is the simulation time interval, $r_{\alpha}^{\text {simulated }}$ is the simulated position of human $\alpha$, and $r_{\alpha}^{\text {tracked }}$ is its real/tracked position. The simulation time interval is kept constant $(T=1.5 \mathrm{~s})$ which is small enough to accurately represent the movement of the corresponding user. The two data points of the simulated trajectories that are taken from the tracked data are the starting point and the desired destination point. The simulation time step, mean speed and variance have been selected based on the real trajectories for each calibration scenario. A summary of the parameters used in the calibration process can be seen in Table I.

$E$ is calculated for all possible combinations for a defined interval of the parameters $A_{\alpha r}$ and $B_{\alpha r}$. An average is determined over the full path to obtain the final value allowing to calculate the fitness level of a particular parameter pair. $E$ is recorded for each combination in Figure 6. The combinations of $A_{\alpha r}$ and $B_{\alpha r}$ with the smallest $E$ are reported as the optimal values and result in local minima in Figure 6. The

TABLE I

A SUMMARY OF THE PARAMETERS USED IN THE PROPOSED MODEL FOR CALIBRATION.

\begin{tabular}{c|ccc}
\hline Parameter & Unit & Assigned value & Reference \\
\hline$v_{\alpha}^{0}$ & $\frac{\mathrm{m}}{\mathrm{s}}$ & $1.25(\sigma=0.1)$ & Data analysis \\
$\tau_{\alpha}$ & $\mathrm{s}$ & $0.20-0.50$ & {$[42]$} \\
$\lambda_{\alpha \beta}$ & - & 0.20 & {$[45]$} \\
$\lambda_{\alpha r}$ & - & 0.20 & Data analysis \\
$r_{\alpha}$ & $m$ & 0.40 & Statistics \\
$r_{r}$ & $m$ & 0.30 & Data analysis \\
\hline
\end{tabular}


travel times and total distances traveled by each agent for all local minima are compared between the simulated and empirical data to find the best combination. The final result of this calibration process shows values of $1.2 \frac{\mathrm{m}}{\mathrm{s}^{2}}$ and $2.6 \mathrm{~m}$ for $A_{\alpha r}$ and $B_{\alpha r}$ respectively with a fitness level of 0.64 .

\section{Validation process}

To validate the calibrated model, human-robot interactions are simulated using $A_{\alpha r}$ and $B_{\alpha r}$ values suggested by the calibration process. The obtained information from simulations (i.e., the position, speed and acceleration at each time step) are then compared to the real data. This is a quantitative assessment which determines the validity of the new model after calibration. A number of scenarios from the humanrobot interaction datasets are selected which are different to the ones chosen for calibration. It is worth noting that the gender and age of the people were not included in the dataset. The results obtained from the validation process (humans speed, acceleration and relative distance error $E$ ) are reported in Table II. The average mean speed of the simulated humans is $1.34(\sigma=0.01) \frac{\mathrm{m}}{\mathrm{s}}$ which compares to $1.25(\sigma=0.02) \frac{\mathrm{m}}{\mathrm{s}}$ for the real ones, a difference of $8 \%$. Acceleration values show a higher difference of $21.78 \%$ comparing the simulated humans $\left(0.23(\sigma=0.07) \frac{\mathrm{m}}{\mathrm{s}^{2}}\right)$ and the recorded human accelerations $\left(0.01(\sigma=0.01) \frac{\mathrm{m}}{\mathrm{s}^{2}}\right)$. The relative distance error $E$ calculated using Equation 8 returns a value of 0.6 which is similar to the relative distance error of the calibration process.

\section{Discussion}

As reported in Figure 4, humans' mean speed in the entire ATC shopping centre is $1.01(\sigma=0.38) \frac{\mathrm{m}}{\mathrm{s}}$. The mean speed of humans in human-robot interaction scenarios is also within a similar range of $1.25(\sigma=0.1) \frac{\mathrm{m}}{\mathrm{s}}$. These values are lower than the average human walking speed (1.35 $\frac{\mathrm{m}}{\mathrm{s}}$ [45]) which is expected. The dataset for this case study was recorded in a closed space within a shopping mall. People inside a shopping mall wander from one shop to another rather than walk single-minded to their destination.

The obtained human-robot interaction strength $A_{\alpha r}$ is $1.2 \frac{\mathrm{m}}{\mathrm{s}^{2}}$ which is in line with the value reported for orthogonal human-robot approaches [28] $\left(1.2 \pm 0.2 \frac{\mathrm{m}}{\mathrm{s}^{2}}\right)$. The value

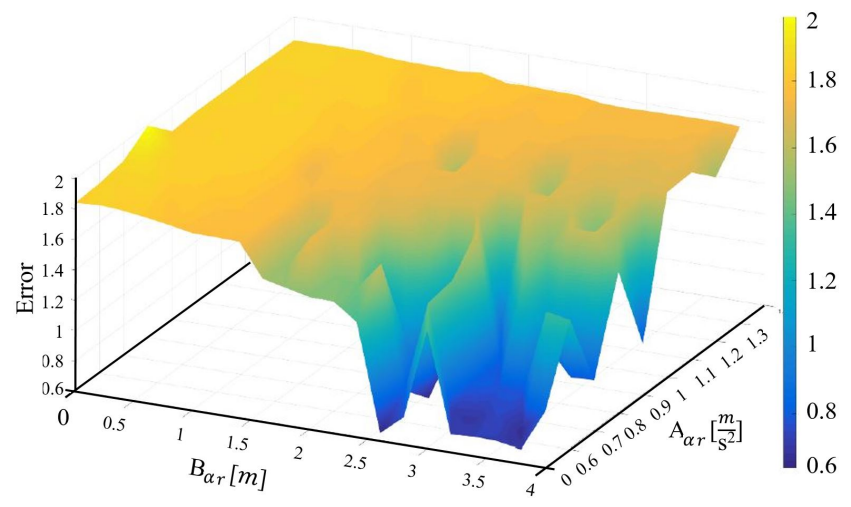

Fig. 6. Fitness surface for parameters $A_{\alpha r}$ and $B_{\alpha r}$. of the interaction range $B_{\alpha r}$ results in $2.6 \mathrm{~m}$ which is within the human-human social zone ranging between 1.2 and $3.6 \mathrm{~m} \mathrm{[43].} \mathrm{However,} \mathrm{this} \mathrm{value} \mathrm{is} \mathrm{larger} \mathrm{than} \mathrm{the}$ human-robot interaction range for teaching tasks $(0.608 \pm$ $0.094 \mathrm{~m})$ [28] and the interaction range for collision avoidance in a small room $(0.46-1.22 \mathrm{~m})$ [44]. The difference in the values for the interaction range can be twofold: It seems that experimental tests in [28], [44] were performed by subjects who are generally familiar with service robots. Humans in the shopping mall might be not familiar with robots which explains the larger interaction range. On the other hand, the preferred personal distance of humans from a robot might vary depending on the type of human-robot interaction (i.e., approaching, teaching, avoiding the robot). The difference between human-robot interaction parameters ( $A_{\alpha r}=1.2 \frac{\mathrm{m}}{\mathrm{s}^{2}}$ and $B_{\alpha r}=2.6 \mathrm{~m}$ ) and human-human interaction values $\left(A_{\alpha r}=0.8 \pm 0.1 \frac{\mathrm{m}}{\mathrm{s}^{2}}\right.$ and $B_{\alpha r}=1 \pm 0.25 \mathrm{~m}$ [32]) highlights the importance of understanding interactions between humans and robots from both sides. The human and robot parameters need to independently calibrated and validated for various types of interactions and scenarios. The relative distance error of 0.6 returned by the calibration process is expected as this model does not only consider orthogonal interaction but human-robot interaction from any angle. In other words, the presented and calibrated model provides a $60 \%$ confidence level of correctly predicting human trajectories when in interaction with a service robot. It should be noted that a higher number of case studies for calibration will result in lower relative distance error and hence an increase in confidence level.

TABLE II

VALUES OF SPEED AND ACCELERATION MEAN AND VARIANCE FOR SIMULATED AND TRACKED PEOPLE

\begin{tabular}{r|cc}
\hline & Simulated people & Tracked people \\
\hline Speed mean $[\mathrm{m} / \mathrm{s}]$ & $1.34(\sigma=0.01)$ & $1.25(\sigma=0.02)$ \\
\hline Acceleration mean $\left[\mathrm{m} / \mathrm{s}^{2}\right]$ & $0.23(\sigma=0.07)$ & $0.01(\sigma=0.01)$ \\
\hline Relative distance error & 0.60 & - \\
\hline
\end{tabular}

\section{CONCLUSIONS}

In this study, HRIs have been modelled based on an extension of the SFM. The proposed model is calibrated and validated using empirical open source data collected from the ATC shopping mall in Osaka (Japan) to understand the socio-psychological interaction of the human in HRIs among crowds. After analysing two datasets and calibrating the key parameters of the new model (interaction range and interaction strength), it becomes evident that humanhuman interactions differ from interactions between humans and service robots. We have shown that humans react to a service robot to a higher extend and within a larger distance compared to the interaction range towards another human. As part of our future work, various methodologies explained in [47] will be used to calibrate and validate the proposed modified SFM for HRIs using different data-sets. Furthermore, the SFM has its limitations as stated in [48], [50], 
[51] and it would be valuable to model HRIs using a velocity dependent SFM and conduct a comparative study.

As discussed in the research domain of Socially-Aware Navigation [12], [49], simple real-time collision avoidance models are not sufficient to assure safe and comfortable HRIs. There is a need for robots to be able to predict humans' future paths and behaviors considering the sociopsychological interaction of the human. Implementing the results of this study could be beneficial to avoid collisions between humans and service robots and, at the same time, to aid the harmonization of robots in public spaces. Further, this model could be used to guide the manoeuvre of driverless cars or pods depending on the location and/or behaviour of the human. Taking into account the human's perspective, this might accelerate the acceptance of autonomous systems to be integrated into the society. It should be noted that the interaction parameters might change over time depending on (i) how familiar the public will become with these autonomous systems, (ii) the type of human-robot interaction (i.e., approaching, teaching, avoiding the robot), and (iii) the type, speed, height and anthropomorphic abilities of the robot.

\section{ACKNOWLEDGMENT}

Many thanks to Ruggero Gargiulo for his contribution to this paper during his Individual Project at the University of Southampton.

The datasets used in this study are available here: http://www.irc.atr.jp/crest2010_HRI/ATC_ dataset/ and http://www.irc.atr.jp/sets/ approach_robot/.

\section{REFERENCES}

[1] R. Murai, T. Sakai, H. Kawano, Y. Matsukawa, Y. Kitano, Y. Honda, K.C. Campbell, A novel visible light communication system for enhanced control of autonomous delivery robots in a hospital, IEEE/SICE International Symposium System Integration, pp. 510516, 2012.

[2] A.G. Ozkil, Z. Fan, S. Dawids, H. Aanes, J.K. Kristensen, K.H. Christensen, Service robots for hospitals: A case study of transportation tasks in a hospital. In Automation and Logistics, IEEE International Conference, pp. 289-294, 2009.

[3] S. Jeon, J. Lee, J. Kim, Multi-robot task allocation for real-time hospital logistics, IEEE International Conference on Systems, Man, and Cybernetics, pp. 2465-2470, 2017.

[4] M.M. Veloso, J. Biswas, B. Coltin and S. Rosenthal, CoBots: Robust symbiotic autonomous mobile service robots, International Conference on Artificial Intelligence, pp. 4423-4429, 2015.

[5] V. Kulyukin, C. Gharpure, J. Nicholson, Robocart: toward RobotAssisted navigation of grocery stores by the visually impaired, IEEE/RSJ International Conference on Intelligent Robots and Systems, pp. 2845-2850, 2005.

[6] D. Kulic, E. Croft, Safe motion planning for human-robot interaction: design and experiments, Mobile Robots: towards New Applications, InTech, pp. 149-170, 2006.

[7] S. Thrun, M. Bennewitz, W. Burgard, A.B. Cremers, F. Dellaert, D. Fox, D. Hahnel, C. Rosenberg, N. Roy, J. Schulte, D. Schulz, MINERVA: A second-generation museum tour-guide robot, IEEE international Conference on Robotics and Automation, pp. 19992005, 1999.

[8] M. Shiomi, T. Kanda, H. Ishiguro, N. Hagita, Interactive humanoid robots for a science museum, IEEE Intelligent Systems, vol. 22(2), pp. 25-32, 2007.
[9] R. Siegwart, K.O. Arras, S. Bouabdallah, D. Burnier, G. Froidevaux, X. Greppin, B. Jensen, A. Lorotte, L. Mayor, M. Meisser, R. Philippsen, Robox at Expo. 02: A large-scale installation of personal robots, Robotics and Autonomous Systems, vol. 42(3-4), pp. 203222, 2003.

[10] W. Burgard, A.B. Cremers, D. Fox, D. Hahnel, G. Lakemeyer, D. Schulz, W. Steiner, S. Thrun, The interactive museum Tour-Guide robot, National Conference on Artificial Intelligence, pp. 11-18, 1998.

[11] H.-M. Gross, H.-J. Boehme, C. Schroeter, S. Mueller, A. Koenig, Ch. Martin, M. Merten, A. Bley, Shopbot: progress in developing an interactive mobile shopping assistant for everyday use, IEEE International Conference on Systems, Man, and Cybernetics, pp. 3471-3478, 2008.

[12] T. Kanda, Enabling Harmonized Human-Robot Interaction in a public space, Human-Harmonized Information Technology, vol.2, pp. 115137, Tokyo, 2017.

[13] M.L. Walters, K. Dautenhahn, R. Te Boekhorst, K.L. Koay, C. Kaouri, S. Woods, C. Nehaniv, D. Lee, I. Werry, The influence of subjects' personality traits on personal spatial zones in a human-robot interaction experiment, IEEE International Workshop on Robot and Human Interactive Communication, pp. 347-352, 2005.

[14] J. Kessler, C. Schroeter, H.-M. Gross, Approaching a person in a socially acceptable manner using a fast marching planner, Intelligent Robotics and Applications, pp.368-377, 2011.

[15] H. Hüttenrauch, K.S. Eklundh, A. Green, E.A. Topp, Investigating spatial relationships in human-robot interaction. IEEE/RSJ International Conference on Intelligent Robots and Systems, pp.5052-5059, 2006.

[16] D. Feil-Seifer, M.J. Matarić, Distance-based computational models for facilitating robot interaction with children, Journal of HumanRobot Interaction, vol. 1(1), pp.55-77, 2012.

[17] K. Dautenhahn, M. Walters, S. Woods, K.L. Koay, C.L. Nehaniv, A. Sisbot, R. Alami, T. Siméon, How may I serve you?: a robot companion approaching a seated person in a helping context, Conference on Human-Robot Interaction, pp. 172-179, 2006.

[18] C. Shi, M. Shiomi, C. Smith, T. Kanda, H. Ishiguro, A Model of distributional handing interaction for a mobile robot, Robotics: Science and Systems Conference, 2013.

[19] C. Shi, M. Shimada, T. Kanda, H. Ishiguro, N. Hagita, Spatial formation model for initiating conversation, Robotics: Science and Systems Conference, 2011.

[20] J.T. Butler, A. Agah, Psychological Effects of Behavior Patterns of a Mobile Personal Robot, Autonomous Robots, vol.10, p.185-202, 2001.

[21] D. Shi, e.g., Collins Jr, B. Goldiez, A. Donate, X. Liu, D. Dunlap, Human-aware robot motion planning with velocity constraints, International Symposium on Collaborative Technologies and Systems, pp. 490-497, 2008.

[22] E. Trulls, A. Corominas Murtra, J. Perez-Ibarz, G. Ferrer, D. Vasquez, J.M. Mirats-Tur, A. Sanfeliu, Autonomous navigation for mobile service robots in urban pedestrian environments, Journal of Field Robotics, vol. 28(3), pp. 329-354, 2011.

[23] P. Trautman, Probabilistic tools for human-robot cooperation, Human Agent Robot Teamwork Workshop HRI, 2012.

[24] B. Kluge, E. Prassler, Recursive agent modeling with probabilistic velocity obstacles for mobile robot navigation among humans, Autonomous Navigation in Dynamic Environments, pp. 121-134, 2007.

[25] A.S. Matveev, C. Wang, A.V. Savkin, Real-time navigation of mobile robots in problems of border patrolling and avoiding collisions with moving and deforming obstacles, Robotics and Autonomous systems, vol. 60(6), pp. 769-788, 2012.

[26] C. Wang, Y. Li, S.S. Ge, T.H. Lee, Adaptive control for robot navigation in human environments based on social force model, IEEE International Conference on Robotics and Automation, pp. 56905695, 2016.

[27] G. Ferrer, A.G. Zulueta, F.H. Cotarelo, A. Sanfeliu, Robot socialaware navigation framework to accompany people walking side-byside, Autonomous robots, vol. 41(4), pp. 775-793, 2017.

[28] Y. Che, C.T. Sun, A.M. Okamura, Avoiding Human-Robot Collisions using Haptic Communication, IEEE International Conference on Robotics and Automation, pp. 5828-5834, 2018.

[29] D. Helbing, A mathematical model for the behavior of pedestrians, Behavioral Science, vol. 36, pp. 298-310, 1991.

[30] B. Anvari, A mathematical model for driver and pedestrian interaction 
in shared space environments, The Universities Transport Study Group (UTSG), 2012.

[31] B. Anvari, W. Daamen, V.L. Knoop, S.P. Hoogendoorn, MGH Bell, Shared space modeling based on social forces and distance potential field, In Pedestrian and Evacuation Dynamics, pp. 907-916, 2014.

[32] B. Anvari, M.G. Bell, A. Sivakumar, W.Y. Ochieng, Modelling shared space users via rule-based social force model, Transportation Research Part C: Emerging Technologies, vol. 51, pp. 83-103, 2015.

[33] B. Anvari, M.G. Bell, P. Angeloudis, and W.Y. Ochieng, Calibration and validation of a shared space model: case study, Transportation Research Record: Journal of the Transportation Research Board, (2588), pp. 43-52, 2016.

[34] B. Anvari, C.K. Nip, A. Majumdar, Toward an accurate microscopic passenger train evacuation model using MassMotion, In 95th Annual Meeting of the Transportation Research Board, vol. 2017. The National Academies Press, 2017.

[35] D. Bršcic, Drazen, T. Kanda, T. Ikeda, T. Miyashita, Person tracking in large public spaces using 3D range sensors, IEEE Transactions on Human-Machine Systems, vol. 43(6), pp. 522-534, 2013.

[36] Y. Kato, T. Kanda, H. Ishiguro, May I help you?: Design of human-like polite approaching behavior, ACM/IEEE International Conference on Human-Robot Interaction, pp. 35-42, 2015.

[37] H. Kidokoro, T. Kanda, D. Bršcic, M. Shiomi, Simulation-based behavior planning to prevent congestion of pedestrians around a robot, IEEE Transactions on Robotics, vol. 31(6), pp. 1419-1431, 2013.

[38] X. Chen, M. Treiber, V. Kanagaraj, H. Li, Social force models for pedestrian traffic-state of the art, Transport Reviews, pp. 1-29, 2017.

[39] K. Lewin, Field Theory in Social Science: Selected Theoretical Papers. New York:Harper \& Row: D. Cartwright (Ed.), 1951.

[40] X. Zhang, Y. Wang, H. Yu, Extended social force model-based mean shift for pedestrian tracking under obstacle avoidance. IET Computer Vision, vol. 11(1), pp. 1-9, 2016.

[41] D. Helbing, P. Molnar, Social force model for pedestrian dynamics. Physical review E, vol. 51(5), p. 4282, 1995.

[42] A. Johansson, D. Helbing, P.K. Shukla, Specification of a microscopic pedestrian model by evolutionary adjustment to video tracking data, Advances in Complex System at World Scientific Publishing Company, vol. 25, 2008.

[43] M.L. Walters, The Design Space for Robot Appearance and behavior for Social Robot Companions, University of Hertfordshire, Dissertation, 2008.

[44] L. Takayama, C. Pantofaru, Influences on proxemic behaviors in human-robot interaction, IEEE/RSJ International Conference on Intelligent Robots and Systems, pp.5495-5502, 2009.

[45] P. Molnár, Modellierung und Simulation der Dynamik von Fußgängerströmen, $\mathrm{PhD}$ thesis, 1995.

[46] G. Ferrer, A. Sanfeliu, Proactive kinodynamic planning using the extended social force model and human motion prediction in urban environments, IEEE/RSJ International Conference on Intelligent Robots and Systems, pp. 1730-1735, 2014.

[47] S. Seer, C. Rudloff, T. Matyus, N. Brändle, Validating social force based models with comprehensive real world motion data, Transportation Research Procedia., Vol. 2, pp. 724-732, 2014.

[48] F. Zanlungo, T. Ikeda, and T. Kanda, Social force model with explicit collision prediction. EPL (Europhysics Letters), vol.93(6), pp.68005, 2011.

[49] J. Rios-Martinez, A. Spalanzani, et C. Laugier, From Proxemics theory to socially-aware navigation: A survey, International Journal of Social Robotics, vol. 7(2), pp. 137-153, 2015.

[50] A. D. Dragan and S. Srinivasa, Generating legible motion, Proc.Robotics: Science and Systems, 2013.

[51] H. Khambhaita et R. Alami, Assessing the social criteria for humanrobot collaborative navigation: A comparison of human-aware navigation planners, 26th IEEE International Symposium on Robot and Human Interactive Communication, pp. 11401145, 2017. ublisher=Springer 